Secondary kinetic deuterium isotope effects.

The CC cleavage of labeled tetramethyl ethylenediamine radical cations - who gets to keep the electron?"

\author{
Christian Bech Nielsen $^{\dagger}$ and Steen Hammerum* \\ Department of Chemistry, University of Copenhagen, \\ Universitetsparken 5, DK-2100 Copenhagen Ø, Denmark
}

\begin{abstract}
The simple cleavage of the CC bond in tetramethyl ethylenediamine molecular ions yields two fragments that are identical but for the positions of the charge and radical; nonetheless, the reactions of deuterium labeled analogs are accompanied by substantial secondary kinetic isotope effects. The underlying transition state zero-point vibrational energy differences depend particularly on the properties of the incipient radicals rather than on those of the charge-retaining products. The $\alpha$-secondary effects arise primarily from vibrations related to deformation of the product $-\mathrm{CH}_{2} \mathrm{~N}$ - and $-\mathrm{CD}_{2} \mathrm{~N}$ - groups when the reactant is labeled at the central $\mathrm{CC}$ bond, whereas $\mathrm{CH} / \mathrm{CD}$ stretching vibrations are the origin of the $\gamma$-secondary effects observed for reactants with labeled methyl groups. These zero-point energy differences are mainly due to hyperconjugative interactions (Bohlmann shifts); the secondary isotope effects on the dissociation of protonated amine dimers have a similar origin.
\end{abstract}

\title{
Introduction
}

Isotope labeling is often used to examine reactions and reaction mechanisms when ions react in mass spectrometers, to provide structural information and to study dynamical aspects of the processes. Kinetic isotope effects arise when the isotopic substitution influences the rate of reactions; the effects are called 'primary' when bonds to the isotopic atom are formed or broken during the reaction, 'secondary' otherwise.

\# $\quad$ Dedicated to the memory of Nico M. M. Nibbering, for many years an important figure in European mass spectrometry research.

+ Present address: Department of Chemistry and Centre for Plastic Electronics. Imperial College London, London SW7 2AZ, UK

* Address for correspondence: steen@kiku.dk 
Kinetic isotope effects on unimolecular gas-phase reactions of ions are generally examined in intramolecular experiments, in studies of symmetrical (apart from the isotopic substitution) reactants. ${ }^{1}$ The first systematic results in this area were reported by Neeter and Nibbering, ${ }^{2}$ who described the secondary kinetic isotope effects that accompany the loss of $\mathrm{CH}_{3}$ and $\mathrm{CD}_{3}$ radicals from the molecular ions of deuterium labeled tert-butylbenzene and tert-butylpyridine (Scheme 1a).

(a)

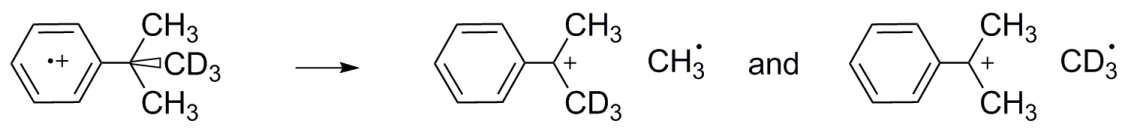

(b)

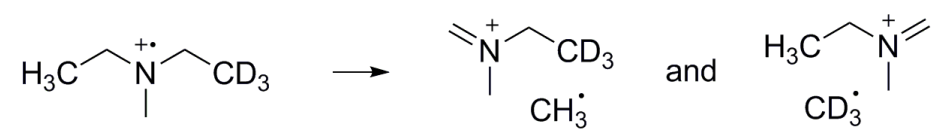

Scheme 1. Typical simple cleavage reactions that are influenced by normal secondary kinetic deuterium isotope effects.

Most reported studies of secondary isotope effects examine the rate of cleavage of two different bonds, but for certain bifunctional molecules the competition involves cleavage of only one bond. One example is the fragmentation of the tetramethyl ethylenediamine (TMEDA) molecular ion (Scheme 2), where cleavage of the central CC bond produces two fragments that are identical except for the location of the charge. The cleavage of deuterium labeled TMEDA molecular ions exhibit remarkable secondary kinetic isotope effects (Figures 1-3).

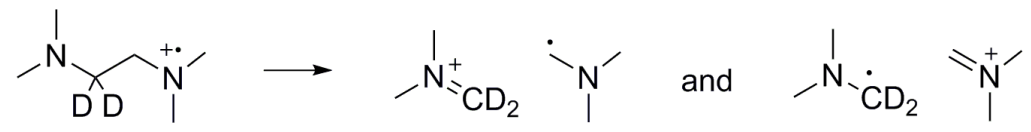

Scheme 2. The cleavage of tetramethyl ethylenediamine- $d_{2}$ molecular ions.

\section{Secondary kinetic isotope effects}

The secondary kinetic isotope effect is defined as the ratio of the rate constants of two near-identical reactions that differ only with regard to isotopic substitution. The origin is nearly always the difference between the transition state zero-point vibrational energies (zpve). The isotope effect is labeled 'normal' if the pathway least likely to be influenced by the isotopic substitution is preferrred. 
The reactions shown in Scheme 1 are influenced by 'normal' isotope effects; loss of $\mathrm{CH}_{3} \cdot$ is in both cases favored over loss of $\mathrm{CD}_{3} \cdot$. The relative yield of the competing reactions is determined by two effects, the zpve-consequences of releasing $\mathrm{CH}_{3}{ }^{\cdot}$ or $\mathrm{CD}_{3}{ }^{\circ}$, and the zpveconsequences of forming a labeled or unlabeled cation. The deuterium substitution gives rise to an $\alpha$-secondary isotope effect on the amine fragmentation where the properties of the incipient methyl radical in particular influence the transition state zero-point vibrational energy. The fragmentation of the tert-butylbenzene ions involves the combination of an $\alpha$-secondary effect (on the loss of methyl) and a $\beta$ secondary effect (on the formation of the benzyl ion).

Isotope effects that give rise to peaks of unequal size in $70 \mathrm{eV}$ electron ionization mass spectra are in most cases difficult to interpret. The ionsource fragmentation involves reactions with rate constants that span five orders of magnitude, and the internal energy of the reacting ions can be correspondingly different. However, field ionization kinetics studies illustrate that statistical weight effects in addition to zpvedifferences can influence pico-second simple cleavage reactions of amine radical cations with deuterium at remote positions. ${ }^{3}$

The reactions of metastable ions, on the other hand, involve reactants with a relatively narrowly delimited internal energy distribution. In particular, the metastable molecular ions that react by straight-forward simple cleavage are those with insufficient internal energy to react rapidly (in the ion source), yet enough to react at all. The metastable ion energy distribution, the 'metastable window', depends on the critical energy of the prominent ion source cleavage reactions.

The metastable peaks that reflect reactions influenced by kinetic isotope effects are conveniently examined with mass analyzed ion kinetic energy spectroscopy (the MIKE technique ${ }^{4}$ ). The ratio of these peaks (heights or areas) represents the ratio of rate constants of the two nearidentical reactions, averaged over the internal energy distibution of the reacting ions, under the assumption that the two rate constants rise equally with increasing internal energy, that the two $k(E)$ curves are parallel.

\section{Variations}

As the ratio of energy-dependent rate constants, the magnitude of the kinetic isotope effect depends on the internal energy of the reacting 
ions ${ }^{5}$ and on the difference between the critical energies of the two reaction channels, that is, on the difference between the transition state zero-point vibrational energies. The isotope effect is further influenced by factors that modify the excess energy of the reacting ions.

The influence of isotopic substitution on the relative rates of simple cleavage reactions with no energy barrier other than the reaction endothermicity can be accounted for with simple RRKM considerations, particularly when comparing isotope effects on the reactions of related metastable ions, or the variation of isotope effects with changed reaction conditions.

Secondary isotope effects are particularly prominent for low-energy reactants. The consequences of small differences between the critical energies of competing reactions become relatively less important if the internal energy increases, and the isotope effect diminishes accordingly.

Among the metastable ions, the higher-energy segment makes the more important contribution to the observed isotope effect because the reaction probability ${ }^{6}$ is a function of internal energy. The higher-energy reactants will show the lower isotope effect, and the isotope effect observed should therefore increase if there is selective depletion of the higher-energy metastable reactant ions. This will be the case in the presence of a third, competing, reaction, which will increase the difference between the yield of the isotopically different reactions (a competitive shift ${ }^{7}$ ). The apparent isotope effect then changes (increases), but the rate constants of the two competing reactions do not.

Reactions with higher critical energy should exhibit lower isotope effects, because the kinetic shift ${ }^{7}$ will allow an increased proportion of higher-energy molecular ions to survive long enough to appear as metastable ions. Likewise, the reactions of larger reactant ions should exhibit decreased isotope effects, because an increased number of degrees of freedom will also result in a kinetic shift and allow more higher-energy molecular ions to reach the mass spectrometer field-free regions. Conversely, the reactions of heavier reactant ions should exhibit an increased isotope effect, as these ions will be older when they reach the field-free regions, as will ions transmitted through the instrument with lower accelerating voltage. 

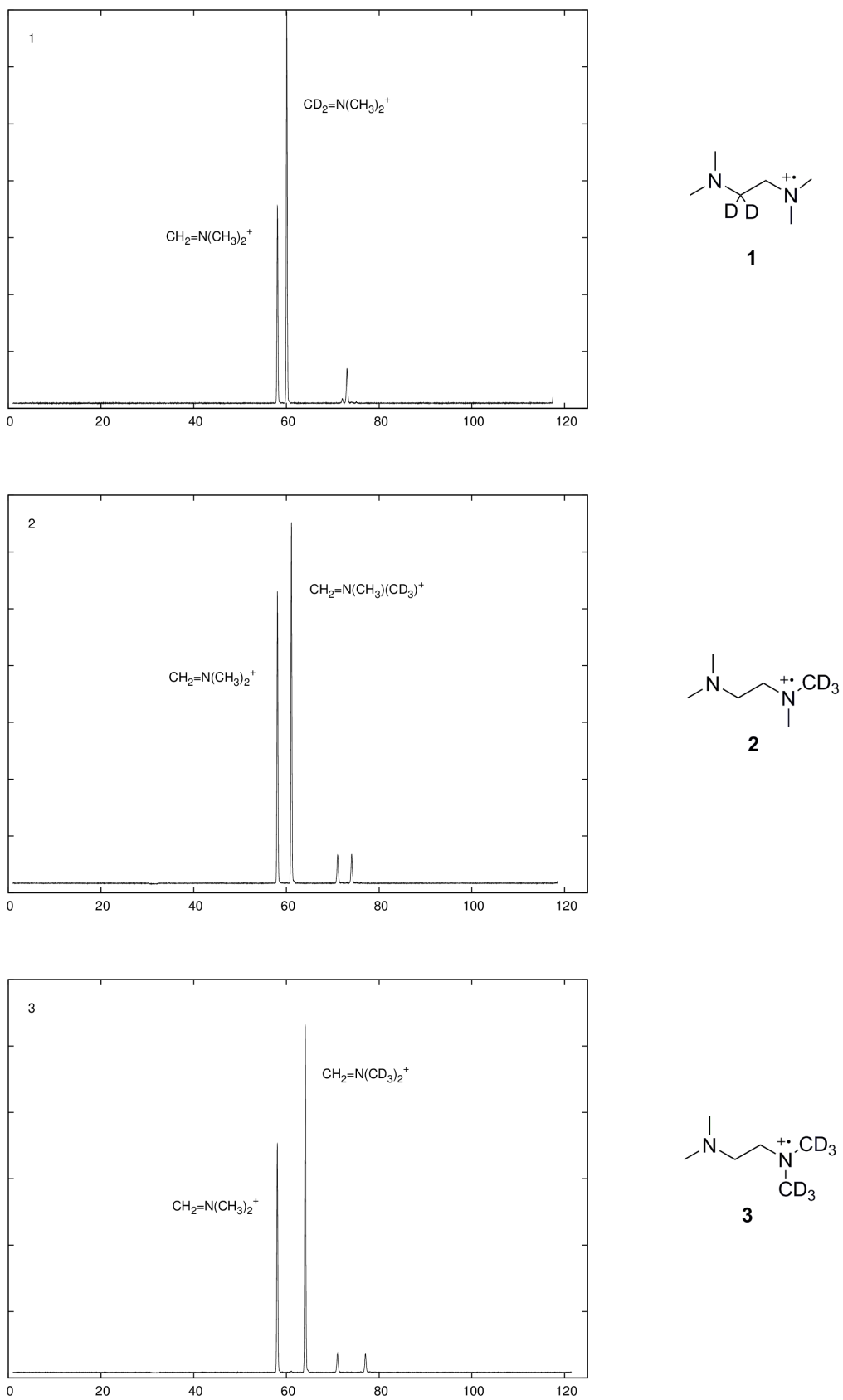

Figures 1-3. MIKE spectra of tetramethyl ethylenediamine molecular ions with 2, 3, or 6 deuterium atoms, respectively. The little peaks arise by loss of $\mathrm{CH}_{3} \mathrm{~N}=\mathrm{CH}_{2}$ neutrals from the molecular ions. 


\section{Instrumental and computational methods}

The spontaneous fragmentation reactions of metastable TMEDA molecular ions formed by $70 \mathrm{eV}$ electron ionization were studied with mass analyzed ion kinetic energy (MIKE) spectrometry. ${ }^{4}$ The MIKE spectra were recorded on a JEOL HX110/HX110A four-sector mass spectrometer (EBEB) operated in three-sector mode. The Gaussian 09 suite of programs ${ }^{8}$ was used to determine structure and harmonic vibrational frequencies with several wave-function and density functional theory methods, employing a variety of basis sets. They all yield qualitatively the same results; typical values in Tables 1-3. Calculations examining the stretching of the TMEDA radical cation CC bond (from 1.5 to $4.0 \AA$ in $0.05 \AA$ increments) were performed with $\mathrm{UMP} 2 / 6-311+\mathrm{G}(\mathrm{d}, \mathrm{p})$. 
Table 1. Calculated harmonic zero-point vibrational energies of the products of cleavage of tetramethyl ethylenediamine radical cations. ${ }^{a}$

$\begin{array}{lllllll}\begin{array}{l}\text { labeled } \\ \text { reactant }\end{array} & \begin{array}{l}\text { labeled } \\ \text { cation }\end{array} & \begin{array}{l}\text { unlabeled } \\ \text { radical }\end{array} & \begin{array}{l}\text { unlabeled } \\ \text { cation }\end{array} & \begin{array}{l}\text { labeled } \\ \text { radical }\end{array} & \Delta \text { zpve }^{\mathrm{b}} & \Delta \text { zpve }^{\mathrm{c}} \\ \mathrm{TMEDA}_{2}(\mathbf{1}) & 275.10 & 281.73 & 292.36 & 265.86 & 1.39 & -0.15 \\ \mathrm{TMEDA}_{2} d_{3}(\mathbf{2}) & 266.35 & 281.73 & 292.36 & 256.05 & 0.34 & 0.34 \\ \text { TMEDA- }_{6}(\mathbf{3}) & 240.32 & 281.73 & 292.36 & 230.36 & 0.67 & 0.69\end{array}$

(a) $\mathrm{kJ} \mathrm{mol}^{-1}$, B2PLYPD/6-311+G(2df,2p), unscaled. $\omega \mathrm{B} 97 \mathrm{XD} / 6-311+\mathrm{G}(2 \mathrm{df}, 2 \mathrm{p})$ results very similar.

(b) Positive values indicate that labeled cation and unlabeled radical is the lowerenergy product combination.

(c) Partial zero-point vibrational energy difference, only vibrations $>2000 \mathrm{~cm}^{-1}$.

Table 2. Harmonic zero-point vibrational energy difference between the products of cleavage of TMEDA- $d_{6}$ radical cations calculated by a variety of methods. $^{\text {a }}$

$\begin{array}{lll}\text { method/basis } & \Delta \text { zpve } & \Delta \text { zpve }>2000 \mathrm{~cm}^{-1} \\ \text { HF/6-31G(d) } & 0.59 & 0.84 \\ \text { B3LYP/6-31G(d) } & 0.59 & 0.82 \\ \text { B3LYP/6-31+G(d,p) } & 0.62 & 0.76 \\ \text { M06-2X/6-311+G(2df,2p) } & 0.55 & 0.60 \\ \omega B 97 X D / 6-311+G(2 d f, 2 p) & 0.65 & 0.69 \\ \text { B2PLYPD/6-311+G(2df,2p) } & 0.67 & 0.69 \\ \text { MP2/6-31+G(d,p) } & 0.48 & 0.68 \\ \text { MP2/6-311++G(d,p) } & 0.48 & 0.66\end{array}$

(a) zpve difference and zpve-contribution to the difference derived from $\mathrm{CH} / \mathrm{CD}$ stretching vibrations over $2000 \mathrm{~cm}^{-1}, \mathrm{~kJ} \mathrm{~mol}^{-1}$, unscaled. 


\section{Tetramethyl ethylenediamine radical cations}

The MIKE spectra in Figures 1-3 demonstrate that secondary kinetic deuterium isotope effects influence the outcome of the simple cleavage of the CC bond of tetramethyl ethylenediamine radical cations (Scheme 2). It makes little sense to label the effects 'normal' or 'inverse', because the competing reactions do not differ with regard to the distance between isotope label and reaction center. One bond is broken, and the outcome is determined by whether charge and unpaired electron are better accommodated in the labeled or unlabeled fragment.

Breaking the $\mathrm{CC}$ bond is expected to be a continuously endothermic simple cleavage. This is confirmed by ab initio calculations describing the stepwise $(0.05 \AA)$ increase of the central CC distance in TMEDA radical cations up to $4 \AA$; a monotonic endothermic reaction. The transition state for reactions close to threshold should then closely resemble the separated products species, ${ }^{9}$ and an estimate of the transition state zero-point vibrational energy can therefore be based on the properties of the cleavage products.

\section{$\alpha$-Labeling, $\mathrm{CH}_{2}$ deformation}

The calculated zpve-differences between the products of cleavage of the $\alpha, \alpha-d_{2}$ labeled TMEDA (1) is approx. $1.5 \mathrm{~kJ} \mathrm{~mol}^{-1}$ (Table 1), favoring the formation of labeled cation and unlabeled radical, in good agreement with the experimental outcome; an isotope effect of $\sim 2.0$ (Figure 1). The predominant contributions to the zpve-difference appear to arise from deformation of the product $\mathrm{NCH}_{2}$ groups, as the stretching vibrations do not contribute significantly to the difference (determined by separating the zpve-contributions from vibrations above and below $2000 \mathrm{~cm}^{-1}$ ). This is in good agreement with the conventional description of $\alpha$-secondary isotope effects on condensed-phase reactions that involve $s p^{3} \rightarrow s p^{2}$ changes. ${ }^{10}$

Both central $\mathrm{CH}_{2}$ groups of TMEDA undergo an $s p^{3} \rightarrow s p^{2}$ change upon cleavage; the conversion to ${ }^{\circ} \mathrm{CH}_{2}-\mathrm{N}$ contributes more to the zpve change than the conversion to $\mathrm{CH}_{2}=\mathrm{N}$. The two $\alpha$-secondary isotope effects that influence the CC-cleavage of tertiary amines ${ }^{11}$ reflect the same two conversions and the resulting similar zpve-differences. 


\section{$\gamma$-Labeling, hyperconjugation}

The zpve-difference between the products of cleavage of TMEDA radical cations with one or two $\mathrm{CD}_{3}$-groups, 2 and 3 , is somewhat smaller, $0.3-0.7 \mathrm{~kJ} \mathrm{~mol}^{-1}$ (Table 1), also favoring the formation of labeled cation and unlabeled radical, in good agreement with the outcome observed experimentally; isotope effects of $\sim 1.26$ and 1.5, respectively (Figures 2 and 3). For these ions, the larger part of the zpvedifference arises from the streching vibrations (determined by examining the zpve-contributions from vibrations above and below $2000 \mathrm{~cm}^{-1}$ ); viewed in isolation, the low wavenumber vibrations would in fact give rise to a reversed isotope effect (Table 2).

The methyl group $\mathrm{CH} / \mathrm{CD}$ stretching vibrations of the incipient neutral dimethylaminomethyl radical exhibit a Bohlmann-shift to lower wavenumbers, ${ }^{12}$ reflecting hyperconjugative interactions between the amino group lone pair and a parallel methyl group $\mathrm{C}-\mathrm{H}(\mathrm{D})$ bond (Scheme 3). The relative zpve-change caused by this shift is not isotopedependent, but the absolute change is greater when the higherwavenumber vibration is involved.

(a)

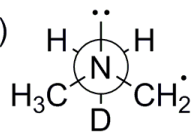

(b)

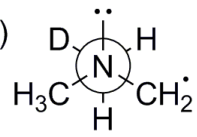<smiles>[AlH][V]</smiles>

Scheme 3. Newman projections of partially deuterium labeled dimethylaminomethyl radicals, illustrating the possible antiperiplanar intractions between lone pair and a methyl group hydrogen/deuterium. The calculated zpve difference favors (b) by $\sim 0.25 \mathrm{~kJ} \mathrm{~mol}^{-1}$ (B2PLYPD/6-311+G(2df,2p)).

The zpve of dimethylaminomethyl radicals with partially labeled methyl groups is therefore lower when $\mathrm{H}$ rather than $\mathrm{D}$ interacts with the lone pair (Scheme 3b). The calculated differences (Table 3) highlight the importance of the orientation of the methyl group $\mathrm{C}-\mathrm{H}$ and $\mathrm{C}-\mathrm{D}$ bonds with respect to the nitrogen lone pair. It follows that in the reactions of 2 or 3 , formation of the unlabeled aminoalkyl radical will be slightly favored. The difference, some $0.3 \mathrm{~kJ} \mathrm{~mol}^{-1}$ for each $\mathrm{CD}_{3}$-group, is sufficient to cause peak ratios considerably different from 1 (Figures 2 and 3). NBO calculations confirm the presence of a strong interaction between lone pair and neighboring anti $\mathrm{C}-\mathrm{H} \sigma^{*}$ orbital in the dimethylaminomethyl radical. The secondary isotope effects that 
accompany dissociation of protonated dimers can be attributed to similarly small zpve-differences ${ }^{13,14}$ (see below).

Table 3. Calculated harmonic zero-point vibrational energy difference between the pairs of products ${ }^{a}$ of cleavage of tetramethyl ethylenediamine molecular ions with deuterium in the methyl groups. ${ }^{b}$

\begin{tabular}{llllll} 
methyl & $\mathrm{H} / \mathrm{D}$ position & $\begin{array}{l}\Delta \text { zpve } \\
\text { (c) }\end{array}$ & (d) & \multicolumn{2}{c}{$\Delta$ zpve $>2000 \mathrm{~cm}^{-1}$} \\
& & & & & $(\mathrm{~d})$ \\
& & 0.25 & 0.21 & 0.21 & 0.19 \\
$-\mathrm{CH}_{2} \mathrm{D}$ & $\mathrm{H}$ anti to lp & -0.01 & -0.03 & 0.03 & 0.02 \\
& D anti to lp & 0.24 & 0.22 & 0.25 & 0.23 \\
$-\mathrm{CHD}_{2}$ & H anti to lp & -0.01 & -0.02 & 0.09 & 0.08 \\
& D anti to lp & 0.34 & 0.31 & 0.34 & 0.33 \\
$-\mathrm{CD}_{3}$ & & 0.67 & 0.65 & 0.69 & 0.67 \\
two $-\mathrm{CD}_{3}$ & & & & &
\end{tabular}
(a) Scheme 2 .
(b) $\mathrm{kJ} \mathrm{mol}^{-1}$; positive value indicates that labeled cation and unlabeled radical is the lower-energy product combination; immonium ions with partially labeled methyl groups have $\mathrm{H}$ eclipsed to the $\mathrm{C}=\mathrm{N}$.

(c) B2PLYPD/6-311+G(2df,2p).

(d) $\omega$ B $97 X D / 6-311+G(2 d f, 2 p)$.

\section{Related systems}

Simple cleavage of a CC bond $\alpha$ to the functional group is a prominent ion-source reaction of most amines and diamines. However, the metastable molecular ions of (substituted) ethylenediamines often undergo rearrangement reactions instead, initiated by intramolecular hydrogen atom abstraction. The reactions in Scheme 4 illustrate the major outcome in a number of simple systems. TMEDA and $N, N, N^{\prime}, N^{\prime}-$ tetramethyl-2,3-butanediamine are exceptions, as their metastable molecular ions fragment nearly exclusively by cleavage of the central $\mathrm{CC}$ bond, possibly because the hydrogen atom affinity of $-\mathrm{NR}_{2}^{+\cdot}$ is 
considerably lower than that of $-\mathrm{NH}_{2}{ }^{+\cdot}$ or $-\mathrm{NHR}^{+}$. Unfortunately, secondary deuterium isotope effects on the cleavage of tetramethyl 2,3butanediamine are not easily studied: extensive scrambling of all methyl group hydrogen atoms precedes simple cleavage.

$\mathrm{H}_{2} \mathrm{~N} \stackrel{+}{\mathrm{N}^{+}} \mathrm{H}_{2} \rightarrow \stackrel{+}{\mathrm{NH}_{2}}+\mathrm{NH}_{3}$<smiles>CNCC[NH2+]C=C[NH2+][CH-]N</smiles><smiles>CC(N)C(C)N</smiles><smiles>CC[NH+]=CC=NC</smiles>

Scheme 4. Simple CC cleavage is not a prominent reaction of most metastable ethylenediamine molecular ions.

The ionized dimethyl ether of ethylene glycol, $\mathrm{CH}_{3} \mathrm{OCH}_{2} \mathrm{CH}_{2} \mathrm{OCH}_{3}$, would be another closely related system, but hydrogen atom abstraction initiates the prominent reactions of the metastable molecular ions also in this case; rearrangement precedes fragmentation. ${ }^{15,16}$ However, Tobita's report ${ }^{15}$ includes a low-resolution MIKE spectrum of a labeled analog, which demonstrates that the low-yield simple cleavage of the CC bond is accompanied by an $\alpha$-secondary isotope effect similar to that described here for TMEDA.

The isotope effects on the dissociation of protonated amine dimers described by Norrman and McMahon ${ }^{14}$ (Scheme 5) are to a large part caused by hyperconjugative interactions in the neutral amine product, closely related to those described above. For dimers with methylamine$d$ and methylamine- $d_{2}$ we find a zpve-difference of $\sim 0.1 \mathrm{~kJ} \mathrm{~mol}{ }^{-1}$ (B2PLYPD/6-311+G(2df,2p)) between neutral product rotamers with $\mathrm{H}$ and $\mathrm{D}$ anti to the lone pair (cf. Scheme 3), in reasonable agreement with the differences calculated for the dissociation of partially labeled TMEDA molecular ions.

$\mathrm{CH}_{3} \mathrm{NH}_{3}^{+}+\mathrm{CD}_{3} \mathrm{NH}_{2} \leftarrow \mathrm{CH}_{3} \mathrm{NH}_{2} \cdots \stackrel{+}{\mathrm{H}} \cdots \mathrm{NH}_{2} \mathrm{CD}_{3} \rightarrow \mathrm{CH}_{3} \mathrm{NH}_{2}+\mathrm{CD}_{3} \mathrm{NH}_{3}^{+}$

Scheme 5. Dissociation of a partially labeled protonated methylamine dimer. Hyperconjugation in the incipient neutral fragments contribute to the zpve differences and in turn to a $\beta$-secondary isotope effect. 


\section{Concluding remarks}

The isotope effects that accompany the $\alpha$-cleavage of tertiary amine radical cations ${ }^{11}$ reflect the change of interactions as the bond breaks, as well as the properties of the incipient immonium ion and (in particular) of the incipient alkyl radical. However, bonding changes in the transition state are not the cause of the $\gamma$-secondary isotope effects that operate when $\mathrm{CH}_{3}$ in TMEDA is replaced by $\mathrm{CD}_{3}$ or when partially labeled protonated methylamine dimers dissociate. In these instances, the zpve-difference reflects the thermochemical and conformational properties of the products rather than the manner of their formation. This will in general be the case for isotope effects on barrier-free simple dissociation reactions when the isotopic substitution is not close to the reaction center. Only $\alpha$ - and $\beta$-secondary effects will be expected to shed light on transition state properties. Condensed-phase conformational isotope effects ${ }^{17,18}$ arise in a similar manner from the thermochemical consequences of zero-point energy differences.

An important contribution to the $\beta$-secondary deuterium isotope effects on the simple cleavage of alkyl groups ${ }^{11}$ is derived from interactions related to the $\gamma$-secondary effect described here. Hyperconjugative interactions between the incipient radical site and vicinal $\mathrm{CH} / \mathrm{CD}$ bonds influence the stretching vibrations ${ }^{19,20}$ and bring about different zpvecontributions from labeled and unlabeled cleavage products.

The deuterium isotope effects on the fragmentation of tetramethyl ethylenediamine molecular ions can be satisfactorily rationalized under the assumption that the transition state closely resembles the final products. Possibly, the isotope effects in this case could be seen as reflecting a charge-exchange equilibrium among otherwise identical fragmentation products, where the electron prefers to stay with the unlabeled species.

\section{Acknowledgement}

The authors gratefully acknowledge productive discussions with Drs. C. B. O. Nielsen, L. F. Østergaard and T. Vulpius. 


\section{Literature references}

1 (a) P. J. Derrick and K. F. Donchi, Mass Spectrometry. In Comprehensive Chemical Kinetics, eds. C. H. Bamford and C. F. H. Tipper, vol. 24; Elsevier, Amsterdam, 1983.

(b) P. J. Derrick, Isotope effects in fragmentation. Mass Spectrom. Rev., 2, 285-98 (1983).

2 R. Neeter and N. M. M. Nibbering, The formation and decomposition of the $[M-\text { methyl }]^{+}$ ion from 4-tert-butylpyridine in comparison with that of the $[M-m e t h y l]^{+}$ion from tertbutylbenzene, as studied by $D$ - and ${ }^{13}$ C-labelling. Org. Mass Spectrom., 7, 1091-1101 (1973).

3 (a) S. Ingemann, E. Kluft, N. M. M. Nibbering, C. E. Alison, P. J. Derrick and S. Hammerum, Time-dependence of the isotope effects in the unimolcular dissociation of tertiary amine molecular ions. Org. Mass Spectrom., 26, 875-81 (1991).

(b) S. Ingemann, S. Hammerum, P. J. Derrick, R. H. Fokkens and N. M. M. Nibbering, Energy-dependent reversal of secondary isotope effects on simple cleavage reactions: tertiary amine radical cations with deuterium at remote positions. Org. Mass Spectrom., 24, 885-88 (1989).

4 R. G. Cooks, J. H. Beynon, R. M. Caprioli and G. R. Lester, Metastable Ions. Elsevier, Amsterdam; 1973.

5 S. Nacson and A. G. Harrison, Dependence of secondary H/D isotope effects on internal energy. Org. Mass Spectrom., 20, 429-30 (1985).

6 S. Hammerum, T. Vulpius and H. E. Audier, Bimodal energy distribution as a consequence of cul-de-sac isomerization reactions. Org. Mass Spectrom., 27, 369 (1992).

7 (a) K. Levsen, Fundamental Aspects of Organic Masss Spectrometry, Verlag Chemie, Weinheim; 1978.

(b) F. W. McLafferty and F. Tureček, Interpretation of Mass Spectra. Univerity Science Books, Sausalito CA; 1993.

8 M. J. Frisch, G. W. Trucks, H. B. Schlegel et al., Gaussian 09. Gaussian Inc, Wallingford CT; 2013.

9 T. I. Sølling, S. Hammerum and T. Vulpius, The influence of fragment size and intermediate barriers on competing near-identical simple cleavage reactions. Int. J. Mass Spectrom., 306, 175-81 (2011).

10 K. C. Westaway, Using kinetic isotope effects to determine the structure of the transition states of $S_{N} 2$ reactions. Adv. Phys. Org. Chem., 41, 217-73 (2006)

11 S. Ingemann, S. Hammerum and P. J. Derrick, Secondary hydrogen isotope effects on simple cleavage reactions in the gas phase: The $\alpha$-cleavage of tertiary amine radical cations. J. Am. Chem. Soc., 110, 3869-73 (1988).

12 (a) F. Bohlmann, Zur Konfigurationsbestimmung von Chinolizin-Derivaten. Angew. Chem. 69, 641-2 (1957).

(b) F. Bohlmann, Lupinen-Alkaloide, VIII. Zur Konfigurationsbestimmung von ChinolizidinDerivaten. Chem. Ber., 91, 2157-67 (1958).

(c) P. J. Krueger and J. Jan, Infrared spectra and the molecular conformations of some amines. Can. J. Chem., 48, 3229-35 (1970).

13 (a) B. D. Nourse and R. G. Cooks, Proton affinity determinations using the kinetic method in an ion trap mass spectrometer. Int. J. Mass Spectrom. Ion Proc., 106, $249-72$ (1991). 
(b) R. A. J. O'Hair, S. Gronert and T. D. Williams, How does isotopic substitution affect the gas-phase proton affinity of glycine? A combined experimental and ab initio study. Org. Mass Spectrom., 29, 151-2 (1994).

(c) F. C. Gozzo and M. N. Eberlin, Primary and secondary kinetic isotope effects on proton and chloronium affinities. J. Mass Spectrom., 36, 1140-48 (2001).

14 K. Norrman and T. B. McMahon, Isotope effects in dissociation reactions of proton bound amine dimers in the gas phase. Int. J. Mass Spectrom., 182, 381-402 (1999).

15 S. Tobita, K. Tonokura and S. Tajima, A study of hydrogen rearrangement reactions in the molecular ion of 1,2-dimethoxyethane by MIKE spectrometry. Mass Spectroscopy, 34, 279-86 (1986).

16 R. Thissen, C. Alcaraz, O. Dutuit, P. Mourgues, J. Chamot-Rooke and H. E. Audier, A hidden hydrogen transfer in the unimolecular reaction of 1,2-dimethoxyethane ${ }^{+}$. J. Phys. Chem. A, 103, 5049-54 (1999).

17 (a) C. L. Perrin, B. K. Ohta and J. Kuperman, $\beta$-Deuterium isotope effects on amine basicity, "inductive" and stereochemical. J. Am. Chem. Soc., 125, 15008-09 (2003).

(b) C. L. Perrin, B. K. Ohta, J. Kuperman, J. Liberman and M. Erdélyi, Stereochemistry of $\beta$-deuterium isotope effects on amine basicity. J. Am. Chem. Soc., 127, 9641-47 (2005).

18 (a) K. T. Greenway, A. G. Bischoff and B. Mario Pinto, Probing hyperconjugation experimentally with the conformational deuterium isotope effect. J. Org. Chem., 77, 9221-26 (2012).

(b) S. Wolfe and C.-K. Kim, A theoretical study of conformational deuterium isotope effects and bond dissociation energies of diastereotropic hydrogens. Can. J. Chem., 69, 1408-12 (1991).

19 J. Pacansky, D. E. Horne, G. P. Gardini and J. Bargon, Matrix isolation studies of alkyl radicals. The characteristic infrared spectra of primary alkyl radicals. J. Phys. Chem., 81, 2149-54 (1977).

20 T. Koenig and R. Wolf, Secondary deuterium isotope effects in radical-forming reactions. J. Am. Chem. Soc., 91, 2574-79 (1969). 